\title{
Real-Time Dashboards to Support eSports Spectating
}

\author{
Sven Charleer, Kathrin Gerling, Francisco Gutiérrez, \\ Hans Cauwenbergh, Bram Luycx and Katrien Verbert \\ Department of Computer Science, KU Leuven \\ Leuven, Belgium \\ \{sven.charleer, kathrin.gerling, francisco.gutierrez, \\ hans.cauwenbergh, bram.luycx, katrien.verbert\}@kuleuven.be
}

\begin{abstract}
eSports matches offer fast-paced entertainment for millions of viewers worldwide, but little is known about how to support a positive viewer experience. One of the key challenges related to popular real-time eSports games (e.g., multiplayer online battle arena games or first-person shooters) is empowering viewers to effectively follow rapid gameplay. In our paper, we address this challenge through the design of dashboards to improve spectator insight and experience in League of Legends, and Counter Strike: Global Offensive. Based on surveys that received a total of 788 responses, we designed information dashboards that we evaluated with 18 experienced eSports viewers. Our results indicate that dashboards contribute to spectator insight and experience, but that careful consideration is necessary to adequately manage in-game complexity and cognitive load of viewers, and establish spectator trust in information dashboards through transparent design. Based on these findings, our paper formulates design goals for spectator dashboards, and outlines key opportunities for future work.
\end{abstract}

\section{CCS Concepts}

-Human-centered computing $\rightarrow$ Information visualization; Graphical user interfaces;

\section{Author Keywords}

Data visualisation; esports; video games; spectator experience

\section{INTRODUCTION}

Gaming live-streams are popular media content where players reunite to watch or support their favourite teams or other professional players, with eSports matches in particular offering fast-paced entertainment for millions of viewers across the world [13]. While the games research community has in depth explored factors that influence the player experience [12] in an abundance of games (e.g. $[10,57]$ ) including titles relevant to eSports [59], it has only begun to address how to design

Permission to make digital or hard copies of all or part of this work for personal or classroom use is granted without fee provided that copies are not made or distributed for profit or commercial advantage and that copies bear this notice and the full citation on the first page. Copyrights for components of this work owned by others than ACM must be honored. Abstracting with credit is permitted. To copy otherwise, or republish, to post on servers or to redistribute to lists, requires prior specific permission and/or a fee. Request permissions from permissions@acm.org.

CHI PLAY 2018, October 28-31, 2018, Melbourne, VIC, Australia.

Copyright (c) 2018 Association of Computing Machinery.

ACM ISBN 978-1-4503-5624-4/18/10 ...\$15.00.

https://doi.org/10.1145/3242671.3242680 for a positive spectator experience [7, 34, 18, 50]. A central element of eSports viewing is making sense of complex, fast-paced gameplay. Modern streaming platforms such as Twitch [29] support this process by allowing streamers to add content on top of their video to augment the viewer experience, for example showing data about the game, or through visual components such as in-game progress, tip-jar, and tip-goals. Likewise, professional eSports streams covering tournaments such as ESL [23] leverage game metrics to facilitate viewer understanding of in-game events.

Inspired by these developments, we explore the design of dashboards offering real-time visualisations of relevant gameplay metrics for eSports spectating, addressing the following research questions: What is the effect of such dashboards on spectator insight (RQ1), and what is the impact of such dashboards on the spectator experience (RQ2)? We focus on two popular eSports titles, the multiplayer online battle arena (MOBA) game League of Legends (LoL) [21], and the first-person shooter (FPS) Counter Strike: Global Offensive (CS:GO) [26], both offering rapid real-time gameplay involving substantial amounts of concurrent action. In our work, we follow a three-stage research process. First, we explore eSports viewers' motives and perspectives on the usefulness of metrics currently available in LoL and CS:GO through online surveys that received a total of 788 responses. Then, we design dashboards for each game drawing from the surveys along with previous work on game analytics and information visualisation. Finally, we carry out a qualitative evaluation with 18 participants to explore the experience facilitated by the dashboards. Our results show that spectators have a strong interest in the dashboard to support their interpretation of gameplay, but also revealed a number of challenges around the cognitive load of eSports viewing, and the establishment of trust when visualising complex data.

Our paper makes three main contributions: first, we provide implementations of dashboards for LoL and CS:GO that contribute to the spectator experience. Second, we formulate design goals that can help researchers and designers in this space wishing to create effective dashboards for eSports settings. Third, we reflect on challenges for dashboard design arising from the complexity of games, and we outline key opportunities for future work. 


\section{RELATED WORK}

In this section, we discuss previous work on spectating in video games, eSports, and visualisations in games to lay the foundation for our research.

\section{Spectating video games}

Spectating video games can happen on both co-located scenarios [55], as a family activity in the living room, an eSports match in a large stadium, or online through mobile devices, personal computers, and smartTVs. Our work focuses on the latter scenario. Twitch and YouTube Live are two of the biggest live video streaming services [40] used by gamers across the globe [51]. These services allow players to stream their game live to millions of viewers. This stream is usually accompanied by audio and/or video of the streamer, commenting on the activities within the game, as well as communicating with their audience who in return can interact with the streamer through integrated chat services. Game spectators are not a passive audience [53]. Lessel et al. [34] for instance explore methods such as voting and drawing on top of the video stream to engage better with the stream and streamer [49]. Cheung \& Huang [7] categorise spectators into multiple personas. "The Entertained" might merely wish to be entertained. The commentator can play an important role: leaving out information to reveal at a later time can create more suspense [7]. "The Inspired" enjoy watching experts and get inspired to play and try similar strategies. Casual streams can foster an informal environment for learning, but competitive streams allow for more advanced players to learn strategies of professional players [47], helping "The Curious" and "The Pupil" who wish to learn from the streams to either improve their knowledge or develop their skills [49].

\section{eSports}

Hamari \& Sjöblom [24] defines eSports as "a form of sports where the primary aspects of the sport are facilitated by electronic systems; the input of players and teams as well as the output of the eSports system are mediated by human-computer interfaces." TL Taylor [54] supports this notion, highlighting that players often compete for substantial financial rewards. eSports is mainly streamed through online services such as Twitch and YouTube [40], where spectators can watch the game and through chat interfaces communicate with fellow spectators. Unlike regular online gaming, eSports usually consists of professional players or teams of players competing against each other, with prize money ranging from hundred thousands to millions of US dollars. Some events are hosted in sports arenas or smaller venues, where opposing players and teams are co-located during matches, allowing fans to spectate and support the "virtual athletes" [30] in the flesh. Certain eSports games manage to fill arenas with over a hundred thousand fans $[22,56]$. Recently, dedicated arenas have been built for the purpose of specific eSports games [14]. The three leading games in eSports are League of Legends (LoL), DOTA 2, and Counter-Strike: Global Offensive (CS:GO) [38].

\section{Visualisations in games}

Visualisations in games can be beneficial for multiple target groups, such as video game developers [61], the player [60], or the game community [2]. Through the Heads-up Display (HUD), games can visualise critical information for the player, such as player's health status, player and quest positions on maps, chat interfaces, and item inventory [45]. In recent years, a new trend attempts to create a better immersion, removing HUD visualisations by integrating critical information into the game world itself, by e.g. visualising health information on the avatar and in-game destination as beacons [35]. Meta-game visualisations such as leaderboards and achievements visually indicate specific game goals and game progress [10], and help compare to other players. Other more elaborate visualisations attempt to give detailed insight post-game into the player and team activities $[37,33,58]$, which in turn can raise awareness of their performance and help rethink strategies. Beyond the player target group, spectators can benefit from game analytics visualisations, such as learning more about the strategies of favourite "e-athletes" or teams, in order to learn and improve their own skills [49]. Visualisations can also assist spectators into getting further insights into the important events during live matches [28]. However, very little is known about how to design visualisations to support spectator experiences.

The focus of this paper is to explore how we can create dashboards for eSports streams with the spectator in mind. We designed and developed dashboards for two of the most popular competitive games, League of Legends and Counter Strike: Global Offensive, in order to understand spectator needs and requirements, the impact of dashboards on their viewing experience, and effect on insight generation.

\section{REAL-TIME VISUALISATIONS FOR ESPORTS VIEWING}

In this section, we explore the development of dashboards to support real-time visualisation of match data in an eSports spectating setting. First, we provide an overview of the games LoL and CS:GO. Then, we report on the general design rationale. We end with a detailed description of the game-specific designs for both the LoL and CS:GO dashboard.

\section{Overview of games \\ League of Legends}

LoL, developed by Riot Games, is a third-person MOBA with multiple game modes, the most popular being "Summoner's Rift", where two teams of five players attempt to destroy each others "Nexus", located in the team's base, which is defended by minions, the opposing team, and turrets. Matches last from 20 to 60 minutes on average. Players control characters called champions, each with a unique set of abilities. Champions start each match at level one and gain experience to level up, unlocking new abilities. Players earn gold by killing nonplayer characters known as minions and monsters, by killing or helping to kill enemy players, and by destroying enemy structures. This gold can be spent on items which boost e.g. champion power or defence. Experience, gold earned, and items are match specific and do not carry over to subsequent matches. Players have a choice of three lanes to reach the opposing team, each lane protected by turrets and spawned minions. Neutral areas between the lanes are the "jungle", home to smaller monsters that can be killed for gold, and larger monsters that require a team effort later in the match. These larger monsters (Elemental drakes, Elder Dragon, Rift 
Herald, Baron Nashor) provide big team boosts when defeated that can alter the course of the match.

During live streams, extra data visualisations are shown additionally to the standard game UI. Most visualisations are part of the video stream: total damage received for each champion during the last fight, total damage received for each champion during the entire game, control wards bought, and baron power gold. The official eSports streaming website also provides an extra sidebar with detailed textual information per champion such as player items, health, gold earned, and minions killed.

\section{Counter-Strike: Global Offensive}

Counter-Strike: Global Offensive (CS:GO) is a multi-player FPS video game, developed by Hidden Path Entertainment and Valve Corporation. Two teams, alternating between Terrorists and Counter Terrorists, compete to plant or defuse a bomb. After each round, players are rewarded based on their individual performance, killing enemy players, and whether or not their team won. The rewards consist of in-game currency to spend on more powerful weapons in subsequent rounds. Competitive mode has two teams of five competing roughly in 45-minute matches, consisting of multiple rounds. The Terrorist team wins when all players in the enemy team are dead or the bomb does not get defused in time and blows up. Counter Terrorists win when all players of the enemy team are dead or the bomb is defused. In contrast to LoL, CS:GO only provides basic information as game overlays, limited to player specific information such as kills, deaths, head-shots, weapon, and money. A second visualisation shows a map which contains the location of players and allows for annotations.

\section{General design rationale}

Bowman et al. [2] provide a visualisation in games design framework with categories to classify visualisation techniques in games: primary purpose, target audience, temporal usage, visual complexity, and immersion/integration. Based on these categories, we first discuss general dashboard design choices.

Temporal usage: Current eSports matches overlay the stream with both continuously present simple status information and occasional intermittent updates regarding recent events, such as damage dealt during a team fight. Similarly, our dashboards provide information at a continuous pace, remaining synchronised with the game.

Visual complexity: Apart from health and mana bars, additional information is usually presented in textual format. However, gamers have a high degree of spatial and visual literacy [4], allowing for more complexity. Our dashboards therefore will provide more information to the spectator in a visual way.

Immersion/integration: Most eSports broadcasters provide information solely as overlays on the video stream. LoL also offers a sidebar containing interactive, textual information. To avoid cluttering the stream, we take a sidebar dashboard approach, similar to the LoL's official sidebar. This space will allow us to provide more elaborate visualisations.

Audience and primary purpose: Our target users are spectators who observe the game.

\section{Survey: Viewer preferences}

To understand viewer preferences specifically relating to the two games, we ran two surveys on reddit.com, targeting r/LeagueOfLegends [43] and r/GlobalOffsensive [42], two of the largest online communities for both games. The survey received 167 responses for LoL and 596 for CS:GO. Furthermore, we explored participants' perceived usefulness for different statistics provided by the respective games to inform the design of the dashboards (LoL: 58 statements integrated in first survey, 167 responses, CS:GO: 37 statements in a separate survey with 25 responses; rated on 5-point Likert scales, 1-Completely disagree, 5-Completely agree), e.g., "I find the gold earned useful while watching a competitive League of Legends match", and "During a round, how important is economy for the outcome of the round?".

The majority of participants were active eSports observers, with more than half of the respondents watching eSports streams at least once a week. Analyzing watching incentives according to the categorisation of Hamari and Sjoblom [24], participants reported most interest towards acquisition of knowledge and enjoyment of witnessing the high skill that players exhibit, reflecting other research into eSports spectator motives [7]. Furthermore, the survey returned insights into the experience related to specific elements of both games.

For LoL, participants found it difficult to keep track of pathing ("It is easy to keep track of jungle pathing (especially early game) in addition to paying attention to the rest of the game aswell". $\mathrm{M}=2.74, \mathrm{~S}=1.18$ ) during the game, however considered jungle pathing important $(\mathrm{M}=4.57, \mathrm{SD}=0.66)$. In terms of match outcome, Gold was assumed relevant $(\mathrm{M}=4.43$, $\mathrm{SD}=0.76$ ), together with game objectives progression such as number of barons $(\mathrm{M}=4.18, \mathrm{SD}=0.83)$ and dragons $(\mathrm{M}=4$, $\mathrm{SD}=0.88)$ killed, and turrets $(\mathrm{M}=4.44, \mathrm{SD}=0.81)$ and inhibitors $(\mathrm{M}=4.23, \mathrm{SD}=0.98)$ destroyed. There is no consensus on whether damage dealt during fights is clear ("It is clear to me how much damage every player is dealing approximately during a team fight." $\mathrm{M}=3.72, \mathrm{SD}=0.99$ and "I'm often surprised by the 'Total damage to champions: last team fight' visualisation" $\mathrm{M}=3.04, \mathrm{SD}=1.23$ ). However, the last team fight damage dealt information that pops up occasionally in the stream is considered important $(\mathrm{M}=4.63, \mathrm{SD}=0.59)$, as is the total damage count $(\mathrm{M}=4.11, \mathrm{SD}=0.95)$. To go beyond simple data visualisations, we asked to rate parameters that could indicate the vulnerability of specific players. The resulting parameters were: distance from team mates $(\mathrm{M}=4.26, \mathrm{SD}=0.9)$, distance from enemies $(\mathrm{M}=4.19, \mathrm{SD}=0.95)$, and defensive summoner spell cool-downs $(\mathrm{M}=4.62, \mathrm{SD}=0.65)$.

In the CS:GO responses, economy was rated most important for outcome of both a round $(\mathrm{M}=4.2, \mathrm{SD}=0.86)$ and the entire match $(\mathrm{M}=4.4, \mathrm{SD}=0.98)$. This led us to visualise both money distribution as well as progression of economy across the entire match. Participants rated "strategy" (e.g. the use of smoke grenades) most important for improving their own knowledge $(\mathrm{M}=4.64, \mathrm{SD}=0.56)$, which a map visualisation could support. There was no consensus on the importance of other statistics; an issue we will further explore through a scoreboard, and visualisation of average damage per round. 


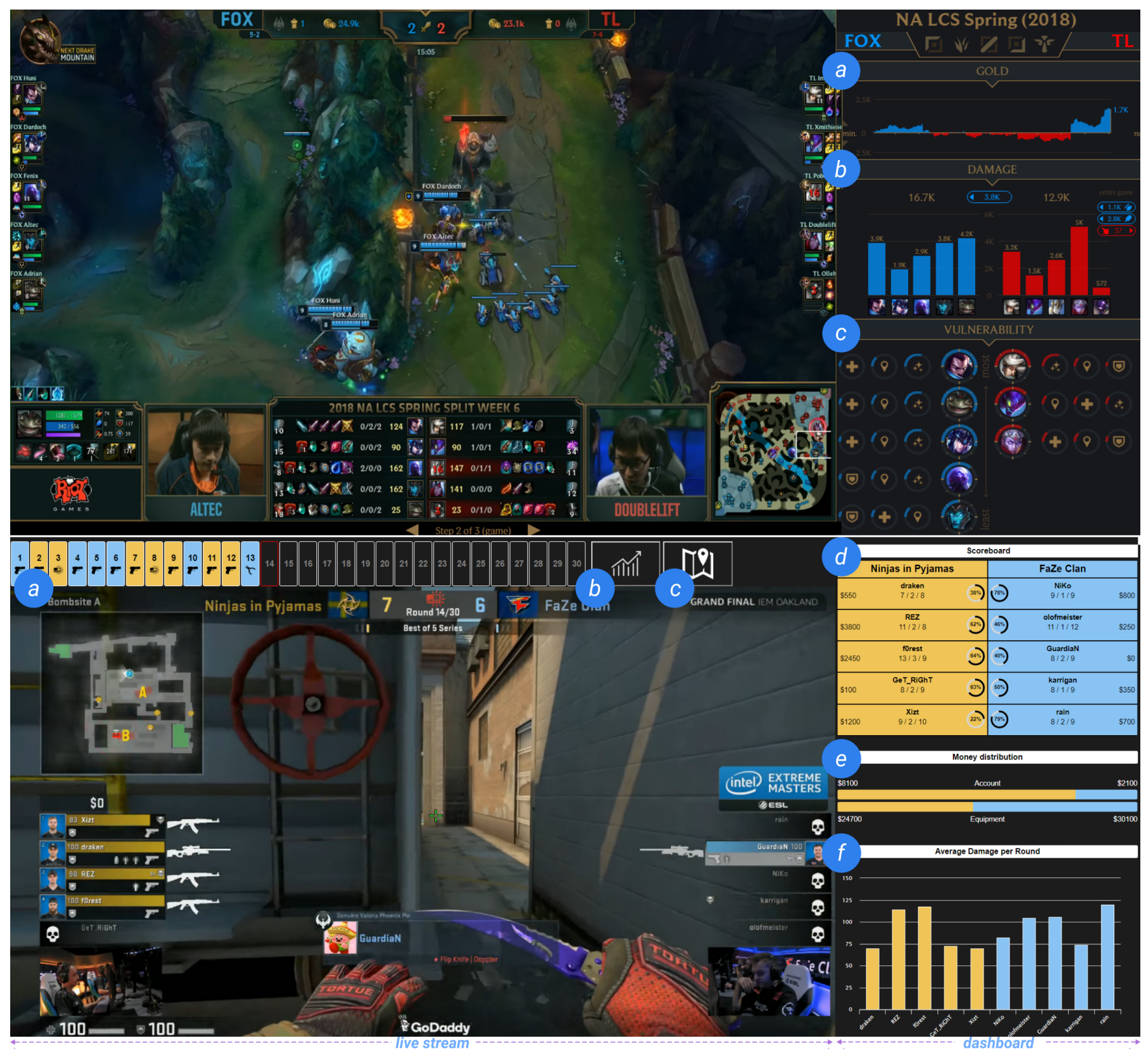

Figure 1. Top: Final LoL dashboard. a) Gold distribution, b) Damage dealt, c) Vulnerability. Bottom: Final CS:GO dashboard. a) Round progression, b) Economy button, c) Map button, d) Scoreboard, e) Money Distribution, f) Average Damage per Round

\section{Game-specific designs}

We followed a user-centred, iterative design approach with intermediate small-scale usability evaluations. In this section, we report on the final iterations of our dashboards for LoL and CS:GO based on the design categories of Bowman et al. [2].

\section{League of Legends dashboard}

Figure 1 (top) presents the dashboard as it is shown next to an official LoL eSports match. It is the result of five iterations: two digital non-interactive designs, two interactive prototypes, and the final fully functioning prototype. Iteration one to four were developed using the interface design tool Figma [17].
Twenty-one participants took part in the evaluations of the iterations of the prototypes (qualitative evaluations: think aloud and semi-structured interview, $\mathrm{N}=8$. Quantitative evaluations: on-line questionnaires, $\mathrm{N}=13$ ).

The main view is split in three modules: Gold, Damage, and Vulnerability. All modules are continuously updated in realtime. Complexity of the visualisations ranges in the intermediate level, providing more detailed information but attempting to keep cognitive load low.

Gold: (Figure 1 top, a) the primary purpose is to provide both status and progression regarding the difference of total gold 


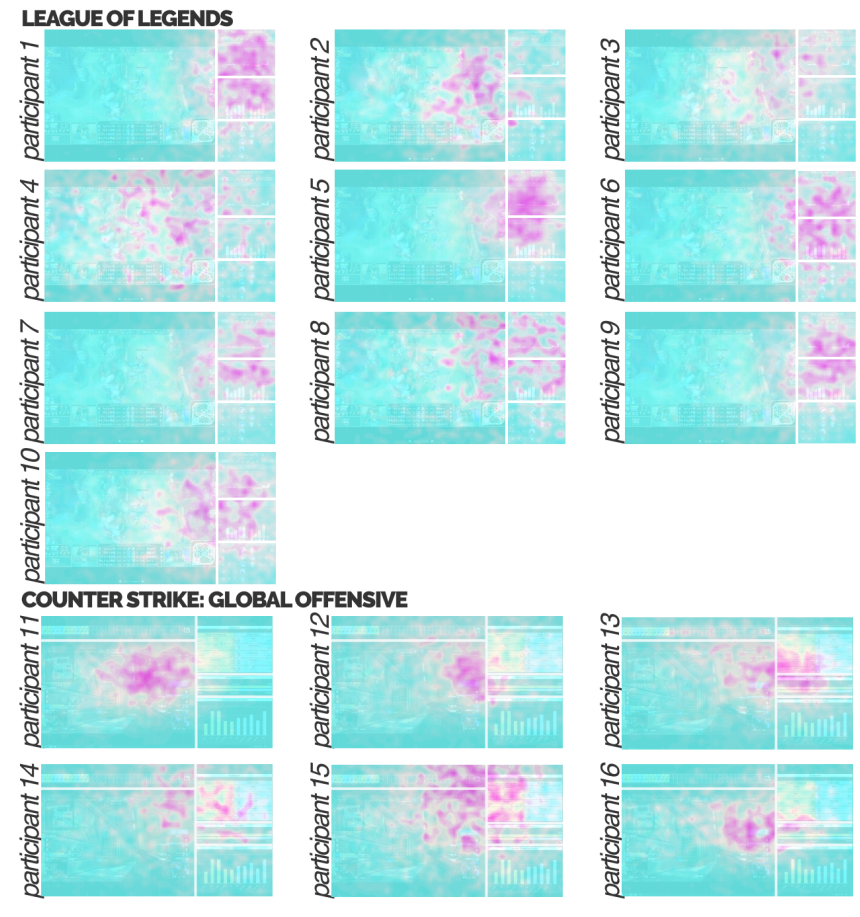

Figure 2. LoL and CS:GO eye tracking results from each participant after the evaluation. The attention heatmap indicates the areas of interest where participants focused more during the whole match.

gathered by both teams over time. The user can switch to a bar chart containing the gold distribution over individual players and their inventory gold (gold amount currently held by a player) and spent gold (providing information regarding the total value of items held by a player).

Damage: (Figure 1 top ,b) the primary purpose is to provide both status and progression of the damage dealt by each player to enemy players. Above the bar charts, the difference in damage dealt between both teams (physical, magic, and true) is summarised. This module allows the spectator to switch between recent damage or damage across the entire match.

Vulnerability: (Figure 1 top, c) provides an indication of how vulnerable each player is. A simple linear equation combines the position disadvantage of the player to the rest of the team and/or enemies, current health and mana points, defensive statistics and summoner spells availability. These values are combined and visualised around the player icon as a summary indicator. This view provides a prospective usage, allowing spectators to make predictions regarding the state of the game.

At the top of the visualisation, the user can filter individual roles to compare e.g. the top-lane players of both teams. When selecting Jungler, the Pathing section appears visualising the recent paths taken by the Junglers.

LoL eSports broadcasters provide a data stream through a Websocket containing detailed information of game events. The dashboard combines and synchronises recorded YouTube matches and the Websocket data and can also be hooked up to live matches that have the Websocket stream available. The dashboard was developed with React.js and D3.js.

\section{Counter-Strike: Global Offensive dashboard}

Figure 1 (bottom) presents the CS:GO dashboard. It is the result of three iterations: a paper prototype, a non-interactive digital prototype designed using Figma, and a fully functioning prototype. Fourteen participants took part in the evaluations of the iterations (think aloud and semi-structured interview). The dashboard consists of Round Progression, Economy, Map, Scoreboard, Money Distribution, and Average Damage per Round. As with the LoL dashboard, all modules are continuously updated in real-time. Complexity of the visualisations are kept at an intermediate level.

Round Progression: (Figure 1 bottom, a) the primary purpose is to provide both status and progression of the 30 match rounds. Played rounds are indicated with the corresponding colour of the winning team and an icon indicating how each round is won (e.g. killing all opponents, detonating bomb, defusing bomb).

Economy: (Figure 1 bottom, b) the Economy button displays a line chart that shows the progression of money owned by each team across rounds at the start of each round.

Map: (Figure 1 bottom, c) the map button overlays a map with the locations of kills, deaths, and all types of grenades used, providing both a continuous and retrospective view.

Scoreboard: (Figure 1 bottom, d) visualises the status of the competing teams and their players. Per player, name, money, kill/assist/death (K/A/D) and head-shot ratio (percentage of head-shot kills) is shown.

Money Distribution: (Figure 1 bottom, e) provides status information regarding total money owned and total equipment value of both teams.

Average Damage per Round: (Figure 1 bottom, f) provides both status and retrospective information by visualising each player's average damage dealt during the match.

The dashboard was created using React and D3.js. The dashboard combines and synchronises CS:GO videos-on-demand (VOD) of matches [16] with demo replay files [27].

\section{EVALUATION}

In this section, we report findings of a qualitative evaluation of the two dashboards with 18 participants, aligned with our overarching research questions focused on the insight and overall experience facilitated by our dashboards.

\section{Method}

We applied semi-structured interviews to gain insights into participants' perspectives on the dashboard. Questions revolved around their spectator experience, insights gained throughout match viewing, and perceived usefulness of the dashboards, e.g., "Did the dashboard change your spectator experience?", "Did you feel that the dashboard changed the insights that you were able to gain from the match?", and "Were there specific elements of the dashboard that stood out to you?". During each evaluation session, we applied eye tracking to gain a 
deeper understanding of how participants interacted with the dashboards. We used WebGazer, an eye tracking library that uses a traditional webcam setup to infer the eye-gaze locations in real time [39].

\section{Participants and Procedure}

Eighteen participants (all male; $\mathrm{N}_{\mathrm{LoL}}=10, \mathrm{~N}_{\mathrm{CS}: \mathrm{GO}}=8$ ) took part in the evaluation. The age range was 22 to 25 . All participants reported above-average experience as eSports spectators in either League of Legends or Counter Strike: Global Offensive. Participants were invited individually to the experiment, and assigned to one of the two conditions (LoL or CS:GO dashboard). At the beginning, participants were given information on the evaluation protocol including eye tracking and audio recording, and were given time to familiarise themselves with the dashboard. Afterwards, participants were shown a video segment of an eSports match where they could freely interact with the dashboard. The LoL segment shown was 22 minutes in length (original match video [15]), the CS:GO segment 26 minutes (original match video [9]). After the match, three short segments of the match were shown again (about $20 \mathrm{sec}-$ onds each), and participants were invited to interact and give feedback. While watching the videos, eye tracking was active. The viewing phase was followed by a semi-structured interview exploring viewer perceptions of the dashboards. At the end of the evaluation, participants provided demographic information. LoL evaluations took on average one hour and 28 minutes, CS:GO evaluations took on average 59 minutes. The entire evaluation session was audio-recorded.

\section{Data Analysis}

Qualitative data were transcribed by the research team and analysed following inductive Thematic Analysis as detailed by Braun and Clarke [3]. Transcripts were thoroughly read and analysed by one researcher, which is in line with the inductive analysis approach that enabled us to freely explore data (for criticism of double coding and inter-coder reliability, please see [19]). We assigned a total of 496 codes that fell into six main themes. For an overview of relative distribution of themes per game/dashboard, please see Table 1.

Eye tracking data were analysed in $\mathrm{R}$. We identified areas of interest: the live stream, the dashboard and the different components in each of the dashboards. We calculated the duration of participant's fixation in all areas of interest over time. Figures 2 and 3 give an overview of the main findings. We further use the data to supplement qualitative findings.

\section{Results}

Theme 1: Complex and Ambiguous Impact of Dashboards on

\section{Spectator Experience}

The most prominent theme (152 codes) addressed the impact of the dashboards on spectator experience, highlighting both instances where the dashboards improved or had a deteriorating effect on spectator experience. In terms of positive instances, common sub-themes focused on additional information provided by the dashboards, their support of further analysis, facilitation of interpretation, and, by extension, supporting learning of the game. For example, participants pointed out that it helped during specific phases of the match, e.g., the buying phase in CS:GO, with one viewer (P15, CS:GO) stating that "You can better understand why they buy things because you can see their equipment and how much money they have". Likewise, LoL viewers highlighted the benefits of the dashboard to further interpret in-game action, e.g., "The vulnerabilities help me understand why junglers choose to gank in certain lanes" (P8, LoL). A dominant theme particularly relevant to viewers of LoL further focused on the potential of the dashboard to manage complexity of gameplay and support viewer understanding of concurrent in-game action. For example, one participant (P10, LoL) outlined the utility of the dashboard to "track the damage in team fights live, because otherwise you have to really keep an eye on who is doing most damage. You don't have time for that." Additionally, participants saw benefits of the dashboard to provide entertainment during slow periods of the match, fill gaps during which viewers were not focused on the game, and to facilitate understanding among less experienced viewers.

However, participants also noted two main negative implications of the dashboard for their experience. Most notably, a number of participants commented that they in fact regarded the dashboard as oversimplification, pointing out that they took pride in their ability of making sense of complex in-game action rather than seeing it as a barrier to a positive experience. For example, "I prefer to analyse such things myself at that right moment during the stream instead of looking at vulnerability. I can deduce it myself from what I see." (P7, LoL). Likewise, participants commented that they did not always see added value of the dashboard over watching the stream only, suggesting that their additional insight gained from the dashboard was minimal.

\section{Theme 2: Need for Lightweight and Flexible Dashboards}

The second prominent theme (138 codes) was concerned with the general design of the dashboards along with viewers' feature preferences. Sub-themes focused on the visual design and placement of the dashboards, automation, ease of access, and utility of included information.

In terms of visual design, participants highlighted appreciation for dashboard designs that blend in with the overall graphical style of the respective game (particularly the LoL dashboard was highlighted in this context). Placement of the dashboard was widely debated with many viewers criticising the amount of screen estate occupied by the boards, and the conflict the sidebar creates when integrated with existing streams, e.g., occluding chat functionality provided by the streaming platform Twitch. In this context, participants highlighted the need for more flexible solutions such as allowing them to choose relevant elements thereby reducing dashboard size, or detaching the dashboard from the main stream to be displayed on a separate screen. Likewise, viewers suggested that automation could support this process, e.g., dynamically determining which elements were relevant at the time of viewing, or automatically closing the dashboard once in-game action picks up: "Damage across the entire game is interesting when not much is going on. It would be nice if the dashboard would automatically switch [to recent damage] when damage is done. Then 

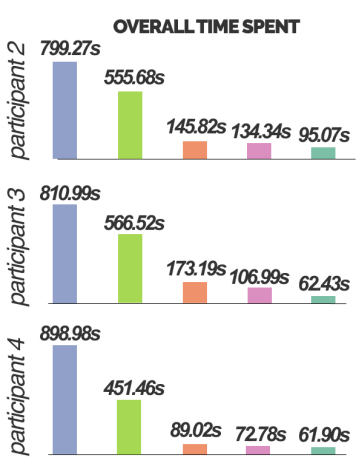

video stream dashboard
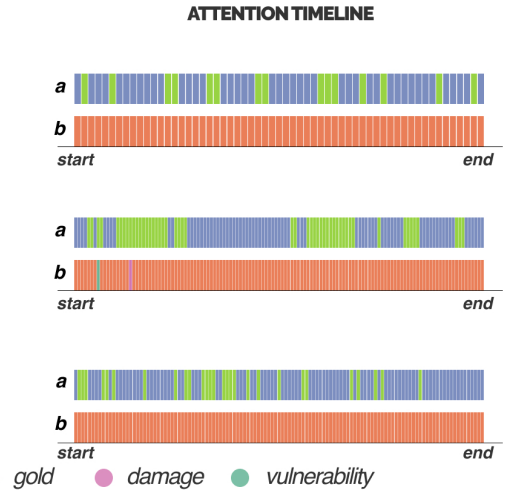
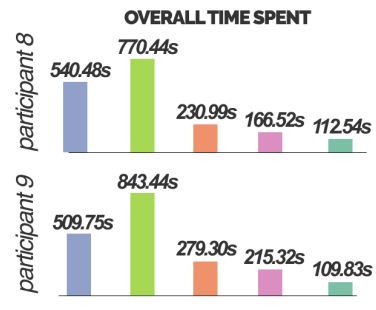

$\frac{1}{2}$
$\frac{\pi}{2}$
$\frac{2}{0}$
$\frac{2}{2}$

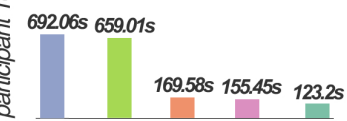

a) stream and dashboard
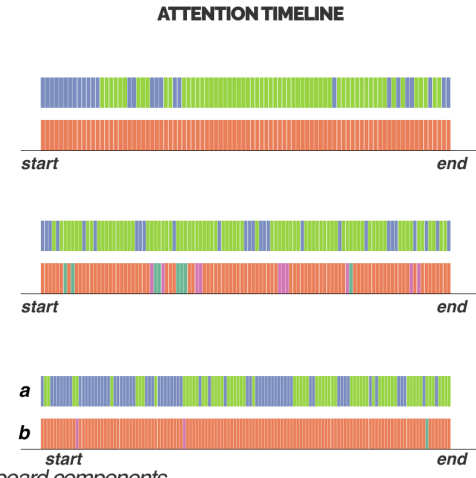

Figure 3. Detailed LoL eye tracking analysis. The overall time spent shows in a bar chart the overall time in seconds participants spent in the areas of interest, and the attention timeline shows the distribution of the attention of the participants during the whole match.

you clearly see when something starts happening. Otherwise you have to constantly manually switch.” (P7, LoL).

Generally, participants highlighted the importance of integrating the dashboards with existing services to facilitate ease of access. In terms of utility of included information, this theme revealed that participants did not appreciate visualisations with existing counterparts (regardless of quality of visualisation). Likewise, particularly simplistic visualisations were regarded as offering superfluous information that could easily be obtained from other sources, or simply not relevant, e.g., "The Round Progression provides a nice overview, but I personally do not think it is very relevant whether they win by killing or detonating." (P17, CS:GO). This suggests an overall preference for more complex visualisations that aggregate a number of easily available metrics, for example, the vulnerability visualisation provided for LoL. Regarding participant preferences for specific dashboard elements, there was little consensus for either LoL or CS:GO, suggesting individual perspectives on elements to be included in real-time visualisations, once more highlighting the need for flexible solutions.

\section{Theme 3: Impact of Dashboards on Cognitive Load}

The tension between spectator experience and the complexity of eSports streaming was further illustrated by another prominent theme (63 codes) addressing the impact of the dashboards on the cognitive load of viewers, with sub-themes focusing on mental and temporal demand, distraction and scaffolding.

It revealed that the dashboards placed additional mental demand on viewers by requiring them to not only pay attention to the video stream, but also track the status of the dashboards: for example, one participant noted that "I think there is too much information [on the dashboard]. Maybe you can personalise certain things." (P3, LoL). Likewise, there were situations in which temporal demand increased, for example, "[It is all going a bit too fast for me], League of Legends is a fast game, sometimes you are not going to be able to follow the game as well. A simple number says enough sometimes." (P7, LoL). As a result, several participants commented that the dashboard at times introduced unwelcome distraction from gameplay, causing them to miss key in-game action and sug- gesting that visualisations can also have a negative impact on spectator experience if they demand too much attention, e.g., "Okay, now I did look a lot [at recent damage] and that did sometimes distract me from the game, I think. Sometimes that caused me to miss an entire team fight" (P1, LoL). In this context, many participants commented on the need for scaffolding particularly to support them when learning to interpret more complex visualisations, e.g., "It is the first time of course, so I think once you start knowing where everything is located, it is going to be easier." (P18, CS:GO). In contrast, other participants commented that the dashboards in fact reduced cognitive load by helping direct their attention toward relevant in-game events, e.g., the beginning of a team fight. However, there were some instances where this effect was undesired, with one participant $(\mathrm{P} 9, \mathrm{LoL})$ commenting that "[Because of the dashboard] I am focusing more on the real data but the skills of the players are not displayed", suggesting that while dashboards can aid understanding of in-game events they might not reflect the full breadth of the viewer experience, e.g., appreciating players' skills.

Figure 2 visualises an aggregated view of the area of interests participants focused on during the entire match. In the case of LoL, four participants (P1,P5,P6,P9) heavily focused on the dashboard; focus in both games was skewed towards the dashboard. This could indicate an impact on the cognitive load (i.e. the dashboard requires more mental effort). Figure 3 details the gaze distribution in time and across the modules of the LoL dashboard. The attention timeline indicates how participants switched focus back and forth between dashboard and game: participants who focused more on the dashboard also focused during longer intervals.

\section{Theme 4: Trust and Complexity of Information}

This theme discusses the ambivalent relationship viewers have with complex visualisations (59 codes), with sub-themes focusing on reliability of information and calculation of metrics.

Throughout analysis, we encountered several instances suggesting that participants did not trust the reliability of information provided by the dashboards. Both viewers of LoL and CS:GO frequently compared dashboard information and 
in-game data to assess correctness, e.g., "I do not really trust it. I saw Kalista running there and she was relatively close to the enemy and suddenly she was most vulnerable while there was only one enemy. So I had a feeling that was not correct." (P1, LoL). Further, participants asked numerous questions around the calculation of metrics; particularly in the case of LoL this caused further discussion as the metrics included in certain visualisations were more complex (e.g., vulnerability) and their construction was not immediately obvious. For example, "The problem is that you can only fully understand it if you know the complete calculation behind it. That remains complex. It would help if you had examples of what typically changes when you enter a fight" (P8, LoL). In some cases, the calculation of metrics was challenged, leading to a devaluation of the entire visualisation. In contrast, there were instances in which viewers expressed appreciation that metrics behind visualisations accurately mapped onto their understanding of game events. [54]

\section{Theme 5: Contextual Factors}

Another theme discovered throughout analysis was concerned with the impact of contextual factors (62 codes) on the utility of the dashboard. Sub-themes focus on a number of relevant aspects; the viewing motive of the spectator, progression of the match, whether the match viewed as a replay or live stream, and the setting it is viewed in: alone, privately with friends, or at a public event. Regarding the viewing motives of spectators, there was large variance, with some participants explicitly commenting that they watched streams to learn from other players and improve their own gameplay, and others stating that they mainly consider watching eSports matches an enjoyable, relaxing leisure activity that they do not wish to be disrupted by excessive focus on game metrics.

Further sub-themes relating to the impact of context on usefulness of the dashboard and its elements were the progression of the match. Many participants commented that the relevance of specific elements of the dashboards depended on the gameplay situation. For example, participants mentioned pathing and vulnerability are interesting when paying attention to the Jungler, while spectators focused on damage dealt during team fights; spectators of CS:GO commented that aspects such as insights into the economy were especially relevant during the buying phase of each round, suggesting that visualisations should flexibly adapt to the stage of play. Regarding the benefits of the dashboards in live or replay situations, participants suggested that obtaining additional insight through reflection would be easier when watching replays. E.g., one participant (P12, CS:GO) commented that "I might prefer to watch later and not during the stream, because I need to be able to think about it", suggesting that the dashboard would be more useful when playback can be paused to facilitating reflection.

Finally, many participants reflected on the impact of the setting in which the match was viewed on the usefulness of the dashboard. While most participants agreed that it would be useful when watching streams individually, there was less consensus on the use of the dashboard in group or public settings. For example, some comments suggested a preference for single viewer use, e.g., "Preferably by myself, because with friends you are less occupied with the details. You will analyse less, so I would do that by myself." (P15, CS:GO). In contrast, other participants felt that the dashboards would improve their experience in live settings regardless of whether they watched individually, in groups, or at public events. In group settings in particular, some participants felt that the dashboards could support analysis and debate, e.g., "Then people see specific things [and you can discuss them]" (P7, LoL).

\section{Theme 6: Usability Challenges for Dashboard Design}

The final theme contains sub-themes directly addressing the usability of both dashboards ( 28 codes). Here, participants highlighted instances in which intuitive interpretation of visualisations was not possible (e.g., displaying health in the LoL visualisations in a circle, but not fully filling it up at full health). Furthermore, participants highlighted further challenges such as possibly poor readability of text on small screens. Likewise, another sub-theme was ease of use, with many participants pointing out that overall usability of both dashboards was good, for example, with one participant (P12, CS:GO) mentioning that "[the dashboard] gives ample indication [of relevant aspects], and interpretation is not cumbersome".

\section{Findings}

Our results demonstrate the utility of real-time data visualisation to support eSports viewing on a general level, but reveal a number of challenges that need to be considered in the design of spectator support tools such as our dashboards. Most importantly, our findings show that viewers naturally consider visualisations most useful if they offer insight beyond what can easily be obtained via already available tools (e.g., information integrated in live streams) or directly derived from gameplay; however, participant feedback also shows that these - often more complex visualisations - are especially problematic in terms of trust and cognitive demand (i.e., viewers showed a need to understand in detail the underlying metrics, and would check correctness to ensure reliability, increasing cognitive load through dashboard use rather than reducing it). More practically, findings from this study show that information dashboards can be designed for different genres of games relevant to eSports, but that careful consideration is necessary to reflect the subtle individual characteristics of each game.

\section{DESIGN GOALS FOR SPECTATOR DASHBOARDS}

Building on our findings, we provide four design goals to help guide researchers and designers wishing to create usable and useful real-time dashboards for eSports viewing. We offer a description of each goal, relate it back to the themes that were crafted during analysis of our evaluation results, and provide examples to illustrate how the goals could be operationalised.

\section{Design Goal 1: Adaptability}

Adaptability of dashboards can facilitate improved spectator experiences. Important aspects of this goal include by allowing viewers to personalise dashboard content according to personal preferences and interests, both in terms of features included (1.1) and level of detail and interpretation of visualised in-game information (1.2). Additionally, the importance of adaptability also applies to the visual appearance of the 
dashboard (1.3); viewers should be free to choose dashboard size and placement.

Related themes. Impact of Dashboards on Spectator Experience - oversimplification; Perspectives on Dashboard Design preferences for specific elements, visual design.

Example. A one-size-fits-all approach is hard to achieve as spectators/gamers have highly individual ideas. Through customisation their needs can be met. Targett et al. [52] suggest that the interface of a videogame is best developed in concert with its players. Their research reports that World of Warcraft [1] customisation is highly focused on managing data that is not immediately visible. Other research fields have found the need for user customisation of dashboards as well $[31,11]$. Similarly, a LoL spectator could detaches the dashboard from the stream to display it on a second screen, choosing to only view aggregate visualisations relating to gold, but no detailed or interpretative elements, allowing them to focus on gameplay over metrics. - A CS:GO spectator views round information as overlay on the stream, and minimises any other dashboard information.

\section{Design Goal 2: Intelligence}

Intelligent dashboards contribute to the spectator experience by generating additional insight by processing complex gameplay metrics, thereby helping to reduce cognitive load of viewers (2.1), by adequately directing viewer attention to relevant elements of play, possibly leveraging predictive models to ensure the dashboard highlights interesting periods of gameplay rather than distracting viewers from it (2.2), and by automatically adapting the presentation of information depending on the ingame situation, both in terms of level of detail of information visualised, and overall visual presentation (2.3).

Related themes. Impact of Dashboards on Cognitive Load - directing attention, distraction, mental demand, temporal demand; Contextual Factors - progression of match, setting.

Example. The dashboard recognises that detonation of the bomb in CS:GO is immediate, and automatically minimises irrelevant dashboard elements (e.g., economy) to allow the viewer to focus on in-game action. - The dashboard notifies the viewer of multiple fights happening in LoL, directing attention to conflicts relevant to overall outcome of the match based on past player performance and indicators of vulnerability.

\section{Design Goal 3: Transparency}

Dashboards offering complex visualisations that include the interpretation of gameplay (such as the vulnerability visualisation included in the LoL dashboard design) need to be transparent about calculations that underlie core visualisations, thereby facilitating learning among viewers (3.1), but more importantly gaining viewer trust (3.2) by easily allowing spectators to compare visualisations to their own interpretation of in-game events and the overall status of the match.

Related themes. Impact of Dashboards on Cognitive Load mental demand, scaffolding; Trust - calculation of metrics, reliability of information.
Example. When presenting visualisations that combine and interpret multiple gameplay metrics, the dashboard offers the opportunity to access additional information on demand that details the elements included, and their contribution to the overall metric. Other fields such as recommender systems have shown that such insight on inner workings of the algorithm can improve acceptance [25]. Drawing from the goal of adaptability, the dashboard also allows expert viewers to adapt the calculation of metrics according to their interpretation.

\section{Design Goal 4: Glanceability}

The real-time requirement of information dashboards for fastpaced eSports environments afford glanceable visualisations that can intuitively be interpreted by viewers, allowing them to maintain focus on in-game action to avoid distraction and reduce cognitive demand on viewers.

Related themes. Impact of Dashboards on Cognitive Load - distraction, mental demand, temporal demand; Usability intuitive interpretation of gameplay visualisations.

Example. Following an "Overview first, zoom and filter, then details-on demand" [46] approach, the dashboard can be limited during periods of fast-paced LoL team fights (see goal Intelligence) to visualise "damage dealt information". This information can be visualised in a high level, at-a-glance, peripheral way following Pousman \& Stasko's [41] design guidelines for ambient displays. The dashboard can still offer the viewer further detail on request.

\section{DISCUSSION}

In our paper, we explore the design of real-time information visualisation tools to support online eSports viewing in two of the most popular games, League of Legends and Counter Strike: Global Offensive. Evaluation results show that the information dashboards that we created are usable and useful to viewers, but that tools in this setting need to be carefully adapted to preferences and needs of spectators. We make these findings actionable through a set of design strategies for real-time data visualisation to support the eSports viewer. In this section, we discuss how dashboards relate to spectator experience and insight in an eSports setting, we reflect on the challenging role of gameplay complexity, and we outline further opportunities for real-time data visualisation in eSports.

\section{Dashboards, Spectator Experience, and Insight}

Information dashboards can be a tool to improve spectator insight (RQ1) and spectator experience (RQ2) in fast-paced online eSports viewing. However, the impact largely depends on the choice of meaningful metrics that needs to be facilitated through a nuanced understanding of the game at hand, along with acknowledgement of diverse viewer motives (e.g., knowledge acquisition and enjoyment of player skill as defined by [24]) which were indicated in initial surveys, but in practice led to instances of conflict (e.g., encouraging focus on metrics rather than highlighting interesting gameplay and facilitating enjoyment). This suggests that a nuanced approach to dashboard design is required, specifically developing solutions that address individual groups of viewers, or creating flexible solutions that can easily be adapted to a range of viewing settings, preferences, and needs. 


\section{The Challenge of Gameplay Complexity in eSports}

A major challenge for data visualisations to support eSports viewers stems from the real-time component and complexity of gameplay, along with managing concurrent gameplay in multiplayer settings that includes fast-paced periods of play.

\section{Cognitive Load and Spectator Experience}

It is tempting to approach the management of cognitive load of viewers arising from the complex nature of eSports in a fashion similar to fast-paced non-entertainment settings as for example car dashboard design for elderly [32], or to take an ambient information visualisation approach [48] where information presented can be important but not critical [41], such as for example classroom settings [5]. However, a point for reflection is the close proximity of the spectator experience and player experience [36], where mastery of challenging and at times complex situations is a core component of a positive experience [44]. Our findings support this notion, showing that managing complexity is a core component of a positive spectator experience, and that oversimplification is detrimental to the experience of some viewers. This suggests that existing approaches toward reducing complexity through visualisation need to be adapted to the eSports setting, asking careful questions around which elements to simplify and where complexity should be maintained.

\section{Reliable and Trustworthy Visualisations}

Ensuring reliability of, and, by extension, achieving spectator trust in visualisations is another core challenge resulting from the need to manage gameplay complexity through interpretative visualisations. This issue has also been addressed by other application areas of data analytics: for example, Chuang et al. [8] discuss the role of interpretation and trust in modeldriven visualisations for text analysis, and propose the principle of progressive disclosure as a means of facilitating understanding through decreasing levels of abstraction, gradually building trust, which is reflected in our design goal of adaptability. Further, they comment on the importance of offering means of model modification, which directly relates to our design goals of transparency and intelligence. In the field of Learning Analytics, Charleer et al. [6] suggest visualising the data "as is", leaving interpretation to expert users based on their tacit knowledge. This aspect is particularly interesting in eSports settings: many viewers are not only spectators but also experienced players [54], suggesting that designers need to create visualisations that accommodate the needs of expert users with in-depth understanding of the underlying mechanics.

Opportunities for Real-Time Data Visualisation in eSports Our work explored the design of dashboards for experienced eSports viewers to improve experience and insight. However, our work revealed a number of further design opportunities for real-time data visualisation for eSports settings. Support tools for live settings. Our results show that intuitive interpretation becomes even more important in live settings, allowing viewers to focus on the stream, while simultaneously providing stimulation for analysis and debate, and supporting access of novice viewers. Support tools for players to analyze performance. Comments showed that viewers are attracted to

\begin{tabular}{lcccc} 
& \multicolumn{2}{c}{ LoL } & \multicolumn{2}{c}{ CS:GO } \\
Themes & Total & \% & Total & $\%$ \\
\hline T1: Impact on spectator experience & 101 & 29.2 & 50 & 33.3 \\
T2: Perspectives on dashboard design & 98 & 28.3 & 39 & 26.0 \\
T3: Impact on cognitive load & 51 & 14.7 & 12 & 8.0 \\
T4: Trust and complexity of information & 54 & 15.6 & 3 & 2.0 \\
T5: Contextual factors & 29 & 8.4 & 32 & 21.3 \\
T6: Usability & 13 & 3.8 & 14 & 9.3 \\
\hline Total & 346 & 100 & 150 & 100
\end{tabular}

Table 1. Relative distribution of codes across themes per game.

analysis tools as something that professional teams rely on, suggesting they would be interested in leveraging it to improve their own gameplay. In-game support to improve performance. Spectators saw benefit of applying visualisations not only as viewers but also to support them in real-time as players, which is a promising opportunity that however also raises concerns around fairness as additional insight into gameplay will of course affect skill levels at which players and teams operate.

\section{LIMITATIONS}

There are a number of limitations that need to be considered when interpreting our findings. Most importantly, we only carried out a small-scale qualitative evaluation based on a single lab session. Our results show that many participants felt a need to learn how to use the dashboards; eye tracking data indicates too much required effort or distraction during live matches, however the novelty of the dashboard must be taken into account; future work should explore dashboard use in a longer-term and possible in-the-wild setting. Furthermore, study participants were exclusively male (which to a certain extent is indicative of the audience [20]); in the future it would be interesting to explore perspectives of more diverse viewer groups. Finally, our work has only begun to explore how dashboards can be integrated to improve the spectator experience in eSports, and only focus on specific games within the FPS and MOBA genres. To address this limitation and produce more generalisable findings, future work needs to explore the design of dashboards for additional games and genres, for example, real-time strategy games which are highly relevant for eSports in Asia.

\section{CONCLUSION}

As eSports viewing turns into a mainstream leisure activity, providing support to ensure positive spectator experiences becomes more important. Our work makes a first attempt to address this challenge by providing real-time dashboards for experienced eSports viewers to support them in their interpretation of gameplay. Findings suggest that such dashboards can support experienced players in their understanding of complex in-game action, and demonstrate the potential of supporting visualisations for other audiences, as for example less experienced spectators. This highlights the opportunity for $\mathrm{CHI}$ PLAY research community to contribute to the further popularisation of eSports viewing through intuitive and informative visualisation of key gameplay elements, thereby increasing the accessibility of the sport. 


\section{REFERENCES}

1. Blizzard Entertainment. 2004. World of Warcraft. Game [PC]. (23 November 2004). Blizzard Entertainment, Irvine, California, U.S.. Last played August 2018.

2. Brian Bowman, Niklas Elmqvist, and T. J. Jankun-Kelly. 2012. Toward visualization for games: Theory, design space, and patterns. IEEE Transactions on Visualization and Computer Graphics 18, 11 (2012), 1956-1968. DOI : http://dx.doi.org/10.1109/TVCG.2012.77

3. Virginia Braun and Victoria Clarke. 2006. Using thematic analysis in psychology. Qualitative Research in Psychology 3, 2 (January 2006), 77-101. DOI : http://dx.doi.org/10.1191/1478088706qp063oa

4. Stuart L Brown. 2009. Play: How it shapes the brain, opens the imagination, and invigorates the soul. Penguin.

5. Sven Charleer, Joris Klerkx, Erik Duval, Tinne De Laet, and Katrien Verbert. 2017a. Towards balanced discussions in the classroom using ambient information visualisations. International Journal of Technology Enhanced Learning 9, 2-3 (2017), 227-253. DOI : http://dx.doi.org/10.1504/IJTEL.2017.084501

6. Sven Charleer, Andrew Vande Moere, Joris Klerkx, Katrien Verbert, and Tinne De Laet. 2017b. Learning Analytics Dashboards to Support Adviser-Student Dialogue. IEEE Transactions on Learning Technologies (2017), 1-1. DOI : http://dx.doi.org/10.1109/TLT.2017.2720670

7. Gifford Cheung and Jeff Huang. 2011. Starcraft from the stands: understanding the game spectator. Proc. of the SIGCHI Conf. on Human Factors in Computing Systems (2011), 763-772. DOI :

http://dx.doi.org/10.1145/1978942.1979053

8. Jason Chuang, Daniel Ramage, Christopher Manning, and Jeffrey Heer. 2012. Interpretation and Trust: Designing Model-driven Visualizations for Text Analysis. In Proc. of the SIGCHI Conf. on Human Factors in Computing Systems (CHI'12). ACM, New York, NY, USA, 443-452. DOI :

http://dx.doi.org/10.1145/2207676.2207738

9. ESL Counter-Strike. 2017. NiP vs. FaZe [Train] Map 3 GRAND FINAL - IEM Oakland 2017. https://www.youtube.com/watch?v=827kgJ0rGHU. (November 2017). Accessed: 2018-08-01.

10. Carlos Cruz, Michael D. Hanus, and Jesse Fox. 2017. The need to achieve: Players' perceptions and uses of extrinsic meta-game reward systems for video game consoles. Computers in Human Behavior 71 (2017), 516-524. DOI : http://dx.doi.org/10.1016/j.chb.2015.08.017

11. Inès Dabbebi, Sébastien Iksal, Jean-Marie Gilliot, Madeth May, and Serge Garlatti. 2017. Towards Adaptive Dashboards for Learning Analytic: An Approach for Conceptual Design and implementation. In 9th International Conf. on Computer Supported Education (CSEDU 2017). 120-131.
12. Alena Denisova, A. Imran Nordin, and Paul Cairns. 2016. The Convergence of Player Experience Questionnaires. In Proc. of the 2016 Annual Symposium on Computer-Human Interaction in Play (CHI PLAY '16). ACM, New York, NY, USA, 33-37. DOI : http://dx.doi.org/10.1145/2967934.2968095

13. Nathan Edge. 2013. Evolution of the gaming experience: live video streaming and the emergence of a new web community. Elon Journal of Undergraduate Research in Communications 4, 2 (2013).

14. Engadget. 2017. Blizzard's first eSports stadium opens for Overwatch. https://www.engadget.com/2017/03/31/ blizzards-first-esports-stadium-opens-for-overwatch/. (March 2017). Accessed: 2018-04-16.

15. LoL Esports. 2018. NA LCS Spring Split : Echo Fox vs. Team Liquid. https://www.youtube.com/watch?v=6sxwo_X59ps. (February 2018). Accessed: 2018-08-01.

16. Eventvods. 2018. Eventvods. https://eventvods.com/featured/csgo. (April 2018). Accessed: 2018-04-16.

17. Figma. 2015. Figma: the collaborative interface design tool. https://www.figma.com/. (December 2015). Accessed: 2018-08-01.

18. Guo Freeman and Donghee Yvette Wohn. 2017. eSports As An Emerging Research Context at CHI: Diverse Perspectives on Definitions. In Proceedings of the 2017 CHI Conference Extended Abstracts on Human Factors in Computing Systems (CHI EA '17). ACM, New York, NY, USA, 1601-1608. DOI: http://dx.doi.org/10.1145/3027063.3053158

19. Dominic Furniss, Ann Blandford, and Paul Curzon. 2011. Confessions from a Grounded Theory PhD: Experiences and Lessons Learnt. In Proc. of the SIGCHI Conf. on Human Factors in Computing Systems (CHI '11). ACM, New York, NY, USA, 113-122. DOI : http://dx.doi.org/10.1145/1978942.1978960

20. Nielsen Games. 2017. U.S. Games 360 Report: 2017. http://www.nielsen.com/us/en/insights/reports/2017/usgames-360-report-2017.html. (October 2017). Accessed: 2018-04-16.

21. Riot Games. 2009. League of Legends. Game [PC]. (27 October 2009). Riot Games, Los Angeles, California, USA. Last played August 2018.

22. Turtle Entertainment GmbH. 2017. Intel and ESL Welcome 173,000 Fans at World's Biggest Esports Event in History.

https://www.eslgaming.com/press/intel-and-esl-welcome173000-fans-world-s-biggest-esports-event-history. (March 2017). Accessed: 2018-07-27.

23. Turtle Entertainment GmbH. 2018. ESL. https://www.eslgaming.com. (April 2018). Accessed: 2018-04-16. 
24. Juho Hamari and Max Sjöblom. 2017. What is eSports and why do people watch it? Internet Research 27, 2 (2017), 211-232. DOI : http://dx.doi.org/10.1108/IntR-04-2016-0085

25. Chen He, Denis Parra, and Katrien Verbert. 2016. Interactive recommender systems: A survey of the state of the art and future research challenges and opportunities. Expert Systems with Applications 56 (2016), 9 -27. DOI : http://dx.doi.org/https: //doi.org/10.1016/j.eswa.2016.02.013

26. Hidden Path Entertainment and Valve Corporation. 2012. Counter-Strike: Global Offensive. Game [PC]. (21 August 2012). Valve Corporation, Bellevue, Washington, USA. Last played April 2018.

27. HLTV. 2018. HLTV. https://www.hltv.org/. (April 2018). Accessed: 2018-04-16.

28. Nate Hoobler, Greg Humphreys, and Maneesh Agrawala. 2004. Visualizing competitive behaviors in multi-user virtual environments. IEEE Visualization 2004 (2004), 163-170. DOI : http://dx.doi.org/10.1109/vISUAL.2004.120

29. Twitch Interactive Inc. 2018. Twitch.tv. http://www.twitch.tv/. (April 2018). Accessed: 2018-04-16.

30. Seth E. Jenny, R. Douglas Manning, Margaret C. Keiper, and Tracy W. Olrich. 2017. Virtual(ly) Athletes: Where eSports Fit Within the Definition of "Sport". Quest 69, 1 (2017), 1-18. DOI : http://dx.doi.org/10.1080/00336297.2016.1144517

31. Min Ji, Christine Michel, Elise Lavoué, and Sébastien George. 2014. DDART, a dynamic dashboard for collection, analysis and visualization of activity and reporting traces. In European Conf. on Technology Enhanced Learning. Springer, 440-445.

32. SeungJun Kim, Anind K. Dey, Joonhwan Lee, and Jodi Forlizzi. 2011. Usability of Car Dashboard Displays for Elder Drivers. In Proc. of the SIGCHI Conf. on Human Factors in Computing Systems (CHI'11). ACM, New York, NY, USA, 493-502. DOI : http://dx.doi.org/10.1145/1978942.1979011

33. Simone Kriglstein, Günter Wallner, and Margit Pohl. 2014. A user study of different gameplay visualizations. Proc. of the 32nd annual ACM Conf. on Human factors in computing systems - CHI '14 (2014), 361-370. DOI: http://dx.doi.org/10.1145/2556288.2557317

34. Pascal Lessel, Alexander Vielhauer, and Antonio Krüger. 2017. Expanding Video Game Live-Streams with Enhanced Communication Channels: A case study. Proc. of the 2017 CHI Conf. on Human Factors in Computing Systems (CHI '17) (2017), 1571-1576. DOI : http://dx.doi.org/10.1145/3025453.3025708

35. Sc Llanos and Kristine Jørgensen. 2011. Do Players Prefer Integrated User Interfaces? A Qualitative Study of Game UI Design Issues. DiGRA 2011 Conf.: Think Design Play (2011), 1-12.

http://citeseerx.ist.psu.edu/viewdoc/download?rep=rep1
36. User Experience Magazine. 2018. Beyond Player Experience: Designing for Spectator-Players. http://uxpamagazine.org/beyond-player-experience/. (February 2018). Accessed: 2018-04-16.

37. B. Medler, B. \& Magerko. 2011. Analytics of Play: Using Information visualisation and gameplay practices for visualising video game data. Parsons Journal for Information Mapping 3, 1 (2011), 1-12. http://piim.newschool.edu/journal/issues/

38. Newzoo. 2017. New Twitch Rankings: Top Games by Esports and Total Viewing Hours.

https://newzoo.com/insights/articles/new-twitchrankings-top-games-esports-total-viewing-hours/. (December 2017). Accessed: 2018-07-27.

39. Alexandra Papoutsaki, Patsorn Sangkloy, James Laskey, Nediyana Daskalova, Jeff Huang, and James Hays. 2016. WebGazer: Scalable Webcam Eye Tracking Using User Interactions. In Proc. of the 25th International Joint Conf. on Artificial Intelligence (IJCAI). AAAI, 3839-3845.

40. Karine Pires and Gwendal Simon. 2015. YouTube live and Twitch. Proc. of the 6th ACM Multimedia Systems Conf. on - MMSys '15 (2015), 225-230. DOI : http://dx.doi.org/10.1145/2713168.2713195

41. Z. Pousman and J. Stasko. 2006. A taxonomy of ambient information systems: four patterns of design. Proc. of the working Conf. on Advanced visual interfaces (2006), 67-74. DOI : http://dx.doi.org/10.1145/1133265.1133277

42. Reddit. 2018a. Counter-strike: Global Offensive subreddit. https://www.reddit.com/r/Globaloffensive. (April 2018). Accessed: 2018-04-16.

43. Reddit. 2018b. League of Legends subreddit. https://www.reddit.com/r/leagueoflegends/. (April 2018). Accessed: 2018-04-16.

44. Richard M. Ryan, C. Scott Rigby, and Andrew Przybylski. 2006. The Motivational Pull of Video Games: A Self-Determination Theory Approach. Motivation and Emotion 30, 4 (Dec. 2006), 344-360. DOI: http://dx.doi.org/10.1007/s11031-006-9051-8

45. Al Dafai Samer. 2016. Conventions within eSports: Exploring Similarities in Design. First International Joint Conf. of DiGRA and FDG 13, 1 (2016), 1-16. http:// www.digra.org/wp-content/uploads/digital-library/paper

46. Ben Shneiderman. 1996. The eyes have it: a task by data type taxonomy for information visualizations. In IEEE Symposium on Visual Languages. IEEE, 336-343. DOI : http://dx.doi.org/10.1109/VL.1996.545307

47. Max Sjöblom, Maria Törhönen, Juho Hamari, and Joseph Macey. 2017. Content structure is king: An empirical study on gratifications, game genres and content type on Twitch. Computers in Human Behavior 73 (2017), 161-171. DOI : http://dx.doi.org/10.1016/j.chb.2017.03.036 
48. Tobias Skog, Sara Ljungblad, and Lars Erik Holmquist. 2003. Between aesthetics and utility: designing ambient information visualizations. In IEEE Symposium on Information Visualization 2003 (IEEE Cat. No.03TH8714). 233-240. DOI : http://dx.doi.org/10.1109/INFVIS.2003.1249031

49. Thomas Smith, Marianna Obrist, and Peter Wright. 2013. Live-streaming Changes the (Video) Game. In Proceedings of the 11th European Conference on Interactive TV and Video (EuroITV'13). ACM, New York, NY, USA, 131-138. DOI : http://dx.doi.org/10.1145/2465958.2465971

50. Samantha Stahlke, James Robb, and Pejman Mirza-Babaei. 2018. The Fall of the Fourth Wall: Designing and Evaluating Interactive Spectator Experiences. International Journal of Gaming and Computer-Mediated Simulations 10, 1 (January 2018), 42-62. DOI : http://dx.doi.org/10.4018/IJGCMS.2018010103

51. Streamlabs. 2017. Streamlabs Live Streaming Report Q2'17 - 53\% Growth, \$100M+, Twitch \& YouTube Crushing It. https://blog.streamlabs.com/streamlabslive-streaming-report-q217-53-growth-100m-twitchyoutube-crushing-it-1b9048efb4e2. (August 2017). Accessed: 2018-04-16.

52. Sean Targett, Victoria Verlysdonk, Howard J Hamilton, and Daryl Hepting. 2012. A study of user interface modifications in World of Warcraft. Game Studies 12, 2 (2012).

53. Nicholas Thiel Taylor. 2016. Now you're playing with audience power: the work of watching games. Critical Studies in Media Communication 33, 4 (2016), 293-307. DOI : http://dx.doi.org/10.1080/15295036.2016.1215481

54. T. L. Taylor. 2012. Raising the Stakes: E-Sports and the Professionalization of Computer Gaming. The MIT Press.
55. Burak S. Tekin and Stuart Reeves. 2017. Ways of Spectating: Unravelling Spectator Participation in Kinect Play. In Proc. of the 2017 CHI Conf. on Human Factors in Computing Systems (CHI'17). ACM, New York, NY, USA, 1558-1570. DOI :

http://dx.doi.org/10.1145/3025453.3025813

56. Time. 2016. I Went to a Massive E-Sports Tournament and Was Blown Away. http://time.com/4543203/leaguelegends-worlds-madison-square-garden/. (October 2016). Accessed: 2018-04-16.

57. Zachary O. Toups, Nicole K. Crenshaw, Rina R. Wehbe, Gustavo F. Tondello, and Lennart E. Nacke. 2016. "The Collecting Itself Feels Good". Proc. of the 2016 Annual Symposium on Computer-Human Interaction in Play CHI PLAY'16 (2016), 276-290. DOI : http://dx.doi.org/10.1145/2967934.2968088

58. Guenter Wallner and Simone Kriglstein. 2016. Visualizations for Retrospective Analysis of Battles in Team-based Combat Games. Proc. of the 2016 Annual Symposium on Computer-Human Interaction in Play CHI PLAY'16 (2016), 22-32. DOI : http://dx.doi.org/10.1145/2967934.2968093

59. Jason Wuertz, Scott Bateman, and Anthony Tang. 2017. Why Players Use Pings and Annotations in Dota 2. In Proc. of the 2017 CHI Conf. on Human Factors in Computing Systems (CHI '17). ACM, New York, NY, USA, 1978-2018. DOI : http://dx.doi.org/10.1145/3025453.3025967

60. Veronica Zammitto. 2008. Visualization Techniques in Video Games. Electronic Information, the Visual Arts and Beyond (2008), 267-276.

61. Georg Zoeller. 2010. Development telemetry in video games projects. https://www.gdcvault.com/play/1012227/ Development-Telemetry-in-Video-Games. (March 2010). Accessed: 2018-04-16. 